\title{
ON THE ENUMERATION OF HOMEOMORPHISM CLASSES OF FINITE TOPOLOGIES
}

\author{
V. KRISHNAMURTHY
}

(Received 1 April 1976)

Communicated by W. D. Wallis

\begin{abstract}
Abst ract
An algorithm called UNLABEL is devised to uniquely label an unlabelled transitive digraph. This is used to construct a one-one correspondence between homeomorphism classes of finite nondiscrete $T_{01}$-topologies and certain generalised Young tableaux of shape-type $\alpha$. For sets of cardinality 2, 3, 4 and 5 these,classes are enumerated and classified in several ways. The notion of a generalised descriptor graph is then introduced to enumerate the homeomorphism classes of all topologies on these sets.
\end{abstract}

\section{Introduction}

Topologies on a finite set $X_{n}=\{1,2, \cdots, n\}$ can be interpreted as transitive digraphs (see Krishnamurthy (1966), Evans, Harary and Lynn (1967)). The counting series for unlabelled transitive digraphs and therefore also for homeomorphism classes of finite topologies is known (see Sharp (1966)) only up to the fifth term and is

$$
x+3 x^{2}+9 x^{3}+33 x^{4}+139 x^{5}+\cdots
$$

But, starting from the time of Davis (1953), who constructed a formula for the number of non-isomorphic reflexive relations, it appears no one has yet published a formula for enumerating the subfamily of transitive relations, which is the family of finite topologies. For $n=2,3$ the problem is easy. For $n=4$ the homeomorphism classes have been enumerated, for $T_{i}$-topologies by Shiraki (1969) and for all topologies by Sharp (1966). Neither of these papers set up, however, an algorithm which can be pursued for higher values of $n$. In this paper we construct a one-one correspondence (see Theorem 1) between homeomorphism classes of non-discrete $T_{0}$-topologies on $X_{n}$ and 
certain generalised Young tableaux (GYT, in short) of shape-type $\alpha$ (for precise formulation, see Sections 1 and 2). The central tool for setting up this correspondence is an algorithm, called UNLABEL, constructed in Section 1, which is a combination of the descriptor graph of Das (1973), the techniques, regarding $T_{0}$-topologies, of Krishnamurthy (1975) and the algorithm INSERT 3 and DELETE 3 of Burge (1974). The latter two algorithms set up a one-one correspondence between certain two-line arrays and GYT of shape-type $\alpha$. The algorithm UNLABEL operates on a GYT of shape-type $\alpha$ and produces a GYT of shape-type $\alpha$. If a GYT is invariant for this operation, we say it is topogeneous. A list of all topogeneous tableaux for $n=4$ (they are 15 in number) is given in Table 2 . The topogeneous tableaux are precisely the ones that correspond to the homeomorphism classes mentioned in Theorem 1. Essentially, the success of our method is in the prescription of a unique labelling of the vertices of an unlabelled transitive digraph.

For a detailed enumeration, however, of the homeomorphism classes, we need to anatomise the above correspondence. In fact we construct (in Theorem 2) a succession of three correspondences (not necessarily one-one) from $T_{n}$-topologies on $X_{n}$ to ordered partitions of $n$ (called level partitions of $n$ ), from ordered partitions of $n$ to formal expressions $k_{\lambda} k_{\mu}$ (which are typical terms of products of homogeneous symmetric expressions), and finally, from such expressions to tableau-shapes $\{\beta\}$ of shape-type $\alpha$. We thus obtain, for $\boldsymbol{n}=2,3,4,5$ the following results. If $C_{n}=\sum N_{\beta}\{\beta\}$ is the counting series for homeomorphism classes of $T_{0}$-topologies on $X_{n}$, with $N_{\beta}$ representing the number of such classes that correspond to the shape $\{\beta\}$, then,

$$
\begin{aligned}
C_{2}= & +\left\{1^{2}\right\} ; \\
C_{3}= & +\left\{1^{2}\right\}+3\left\{21^{2}\right\}, \text { from Table } 1 ; \\
C_{4}= & 1+\left\{1^{2}\right\}+4\left\{21^{2}\right\}+6\left\{31^{3}\right\}+2\left\{2^{3}\right\} \\
& +2\left\{32^{2} 1\right\}, \text { from Table } 3 ; \text { and } \\
C_{5}= & +\left\{1^{2}\right\}+4\left\{21^{2}\right\}+7\left\{31^{3}\right\}+4\left\{2^{3}\right\}+17\left\{32^{2} 1\right\} \\
& +12\left\{41^{4}\right\}+7\left\{3^{2} 2^{2}\right\}+5\left\{42^{2} 1^{2}\right\} \\
& +1\left\{3^{4}\right\}+4\left\{432^{2} 1\right\}, \text { from Table } 5 .
\end{aligned}
$$

We thus also recover the counting series for the number of $T_{0}$-topologies on $X_{n}$ as

$$
2 x^{2}+5 x^{3}+16 x^{4}+63 x^{5}+\cdots \cdots
$$

known to Sharp (1966). 
Recall (see Theorem 3 of Stanley (1977)) that once the number of homeomorphism classes of $T_{0}$-topologies of all orders up to $n$ is known, it is possible to find the number of homeomorphism classes of all topologies of order $n$. In order to enumerate them, however, we introduce the concept of a generalised descriptor graph. Using this and the Table 2 of Krishnamurthy (1975), we give, in Tables 6 and 7 of Section 6, a detailed enumeration and classification of the homeomorphism classes of all non- $T_{0}$-topologies up to order 5, thereby also resulting in the counting series (1). Incidentally, this confirms that the counting series of Harary and Palmer (1962), (page 218, $\mathbf{P 2 . 4 )}$ is in error.

The detailed classification, in terms of $k_{\lambda} k_{\mu}$ and the descriptor graphs, that is presented in Table 4 for the homeomorphism classes of non-discrete $T_{0}$-topologies on $X_{5}$ and the summaries presented in the other tables, in terms of tableau-shapes and partitions, contain possibly more information than what this paper has attempted to read from them. A generating function for the series (2) perhaps lies hidden in these tables, but the author does not yet know how to construct it.

\section{Topogeneous tableaux}

A generalised Young tableau, shortly, a GYT, or still briefly a tableau, of shape $\left\{p_{1} p_{2} \cdots \cdots p_{m}\right\}$ with $p_{1} \geqq p_{2} \geqq \cdots \geqq p_{m} \geqq 1$ is an arrangement of positive integers in $m$ rows with $p_{i}$ entries in the $i$ th row, with the property that the entries are non-decreasing from left to right in each row and strictly increasing from top to bottom in each column. The shape can also be described in Frobenius notation as

$$
\begin{array}{llll}
a_{1} & a_{2} & \cdots & a_{r} \\
b_{1} & b_{2} & \cdots & b_{r}
\end{array}
$$

where $a_{i}$ is the number of entries to the right of the $i$ th element of the leading diagonal and $b_{i}$ is the number of entries below it. If the shape of a tableau is expressible in Frobenius notation as any one of the forms

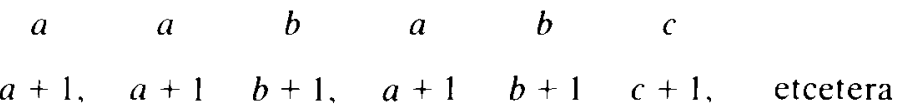

where the $a$ 's, $b$ 's, $c$ 's $\cdots$ are non-negative integers, we say the tableau is of shape-type $\alpha$. Note that the shapes that occur in the expressions for $C_{2}, C_{3}, C_{4}$ and $C_{5}$ in (2) are all of shape-type $\alpha$.

Any finite set of tableaux shall be considered to have been sequenced by the 'first letter sequence' defined as follows. Fill each of these tableaux with 
sufficient number of zeros so that all the tableaux, thus extended, have the same number of rows and columns. Read each such extended tableau as one string of numbers by reading it row after row in continuation. Sequence these strings of numbers lexicographically according to the natural order among the numbers $\{0,1,2, \cdots, n\}$. For example, the set

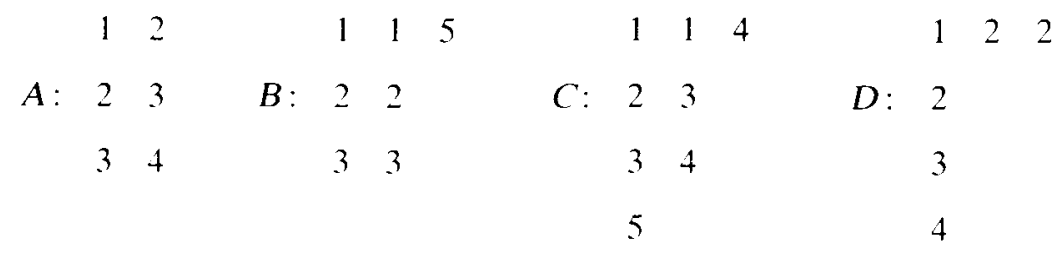

would be sequenced as $C, B, A, D$.

We shall also be considering two-line arrays

$$
\begin{array}{llll}
u_{1} & u_{2} & \cdots & u_{d} \\
v_{1} & v_{2} & \cdots & v_{d}
\end{array}
$$

where the $u$ 's and $v$ 's are positive integers. The two-line array is said to be in standard form if

(i) $u_{k}>v_{k}$ for all $k$;

(ii) $u_{k} \leqq u_{k}$, for all $k$; and

(iii) $u_{k}=u_{k, 1} \Rightarrow v_{k}>v_{k, 1}$.

All our two-line arrays shall be taken to be in standard form, unless otherwise mentioned or constructed.

An algorithm of Burge (1974) known as INSERT 3 converts such two-line arrays into tableaux of shape-type $\alpha$. This algorithm and its inverse DELETE 3 depend on a knowledge of the algorithms INSERT and DELETE of Knuth (1970).

A tableau whose parts belong to $\{1,2, \cdots, n\}$ and include 1 and $n$ shall be called a tableau of order $n$. We shall now define an algorithm called UNLABEL which operates on a tableau $A$ of order $n$ and shape-type $\alpha$ and ultimately produces a tableau $B$. also of order $n$ and shape-type $\alpha$.

UNLABEL:

Step 1. Using DELETE 3. convert $A$ into a two-line array

$$
\Gamma: \begin{array}{lllll} 
& u_{1} & u_{2} & \cdots & u_{m} \\
& v_{1} & v_{2} & \cdots & v_{m}
\end{array}
$$

Burge (1974) shows that this will be in standard form.

Step 2. (Removal of Transitivity). Form the sets $T(\alpha)=\left\{v_{k}: u_{k}=\alpha\right\}$ for 
each $\alpha=n, n-1, \cdots, 3$. Start with $T(n)$. Check, whether, for $x, y \in T(n)$, with $x>y$, there is, in $\Gamma$, a chain of pairs

$$
\left(u_{i}, v_{i}\right),\left(u_{i}, v_{j}\right), \cdots, \quad\left(u_{k}, v_{k}\right),\left(u_{l}, v_{l}\right)
$$

such that

$$
x=u_{i}, \quad v_{i}=u_{j}, \quad \cdots, \quad v_{k}=u_{i} \quad \text { and } \quad v_{l}=y .
$$

If so, remove the pair $(n, y)$ from $\Gamma$. Do this for every $x, y \in T(n)$ with $x>y$. Repeat the process for $T(\alpha)$, with $\alpha=n-1, n-2, \cdots, 3$. Call the resulting array

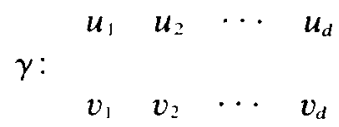

Step 3. (Level partition). Write $U(\gamma)$ (respectively, $V(\gamma)$ ) for the set of integers from 1 to $n$ that occur among the $u$ 's (respectively, $v$ 's) of $\gamma$. With $\gamma_{0}$ standing for $\gamma$, recursively define sets $S_{i}(\gamma), i=1,2, \cdots, l$ and 'truncated' arrays $\gamma_{i}, i=1,2, \cdots, l-1$ as follows:

$$
\begin{gathered}
S_{1}(\beta)=\{1,2, \cdots, n\} \backslash U(\beta) \text { for any array } \beta \\
S_{i}(\gamma)=S_{1}\left(\gamma_{i-1}\right) \backslash S_{1}\left(\gamma_{i-2}\right), i=2, \cdots, l
\end{gathered}
$$

and for $i=1,2, \cdots, l-1, \gamma_{i}$ is the array that remains after the removal of those pairs $\left(u_{k}, v_{k}\right)$ from $\gamma_{i-1}$ for which $v_{k} \in S_{i}(\gamma)$. Note that $l$ is to be determined so that $\gamma_{t-1}$ is the trivial array without any pairs and $\gamma_{t-2}$ is non-trivial. Finally,

$$
S_{1}\left(\gamma_{l-1}\right)=\{1,2, \cdots, n\}
$$

Thus we have an ordered decomposition of the set $\{1,2, \cdots, n\}$ into $l$ sets $S_{i}(\gamma), i=1,2, \cdots, l$ with $\left|S_{i}(\gamma)\right|=p_{i}$, say. $\left(p_{1} p_{2} \cdots, p_{l}\right)$ is an ordered partition of $n$. We shall call it a 'level partition' of $n . S_{i}(\gamma)$ is said to be the set of 'points' at level $i$.

Step 4. (Ordering the level decomposition). Rename the set $S=$ $\{1,2, \cdots, n\}$ by a suitable permutation of $S$ and form a revised array $\delta$, in standard form, such that the ordered set

$$
\left\{S_{1}(\delta), S_{2}(\delta), \cdots \cdots S_{1}(\delta)\right\}
$$

satisfies

$$
S_{i}(\delta)=\left\{\zeta_{i-1}+1, \zeta_{i 1}+2, \cdots, \zeta_{i-1}+p_{i}\right\}
$$

where 


$$
\zeta_{i}=\sum_{k=1}^{\prime} p_{k}, \quad i=1,2, \cdots, l \quad \text { and } \quad \zeta_{0}=0 \text {. }
$$

The array $\delta$, with its level paritition is called a descriptor array.

Step 5. (Construction of symmetric expression). Construct the formal expression:

$$
\prod_{i} x_{i}^{\alpha_{i}} \quad \prod_{i} y_{i}^{\beta_{1}}
$$

where $x_{i}$ and $y_{i}$ stand for the integer $i$ appearing in $\delta$; and $\alpha_{i}$ (respectively, $\beta_{i}$ ) is the number of times $i$ appears among the $v$ 's (respectively, $u$ 's) in $\delta$.

Step 6. (Towards standardization of symmetric expression). Check whether (4) has the property:

$$
*\left\{\begin{array}{l}
\text { At any level } t \\
i>i^{\prime} \Rightarrow \alpha_{i} \leqq \alpha_{i^{\prime}} ; \quad \text { and } \\
i>i^{\prime}, \quad \alpha_{i}=\alpha_{i^{\prime}} \Rightarrow \beta_{i} \leqq \beta_{i^{\prime}} .
\end{array}\right.
$$

If not, renumber the 'points' within the same level suitably so that $(*)$ is ensured. Call the so renumbered array (again put in standard form) $\delta^{\prime}$.

Step 7. (Check for ambiguity in standardization). Check whether, in $\delta^{\prime}$, at any level $t$, there are points $i$ and $i^{\prime}$ such that $\alpha_{i}=\alpha_{i}$ and $\beta_{i}=\beta_{i}$. If there is none, go to step 9. Otherwise, go to step 8.

Step 8. (All possible choices for the ambiguities). Form all the sets $S_{i}(\alpha, \beta)=\left\{i\right.$, at level $t: \alpha_{i}=\alpha$ and $\left.\beta_{i}=\beta\right\}$ for various levels $t$ and for possible values $\alpha, \beta$. For every possible reassignment of numbers to the points of the sets $S_{1}(\alpha, \beta)$, revise the array $\delta^{\prime}$ and put it in standard form.

Step 9. (Unique choice of tableau). Using INSERT 3, convert $\delta^{\prime}$ and each of the revised arrays, if any, obtained in step 8, into tableaux of shape-type $\alpha$. Among the tableaux so obtained, choose the earliest one, according to the 'first letter sequence', and call it $B$.

Definition 1. The result of applying UNLABEL on $A$ is $B$.

EXAMPLE. Let us apply the algorithm on

$\begin{array}{lllll} & 1 & 1 & 2 & 3 \\ & 2 & 2 & 3 & \\ & 3 & 4 & 4 & \\ & 5 & 6 & 6 & \\ & 6 & & & \end{array}$


We only indicate the results after each step.

Step 1.

$$
\begin{aligned}
& \begin{array}{lllllll}
2 & 3 & 4 & 5 & 6 & 6 & 6
\end{array} \\
& \Gamma \text { : } \\
& \begin{array}{lllllll}
1 & 2 & 1 & 3 & 4 & 3 & 2
\end{array}
\end{aligned}
$$

Step 2.

$$
\begin{aligned}
& \begin{array}{llllll}
2 & 3 & 4 & 5 & 6 & 6
\end{array} \\
& \gamma: \\
& \begin{array}{llllll}
1 & 2 & 1 & 3 & 4 & 3
\end{array}
\end{aligned}
$$

Step 3.

$$
\begin{aligned}
& S_{1}(\gamma)=\{1\}, \quad S_{2}(\gamma)=\{2,4\}, \\
& S_{3}(\gamma)=\{3\} \text { and } S_{4}(\gamma)=\{5,6\} .
\end{aligned}
$$

Step 4.

$$
\begin{array}{cccccccc}
\delta: & 2 & 3 & 4 & 5 & 6 & 6 \\
S_{1}(\delta)=\{1\}, S_{2}(\delta)=\{2,3\}, S_{3}(\delta)=\{4\} & & 1 & 1 & 2 & 4 & 4 & 3
\end{array}
$$

\section{Step 5.}

$$
x_{1}^{2} x_{2} x_{3} x_{4}^{2} y_{2} y_{3} y_{4} y_{5} y_{6}^{2}
$$

\section{Step 6.}

After an interchange of 5 and 6 we get

$$
\begin{array}{lllllll} 
& 2 & 3 & 4 & 5 & 5 & 6 \\
\delta^{\prime}: & 1 & 1 & 2 & 4 & 3 & 4
\end{array}
$$

\section{Step 7.}

There is ambiguity at level 2 ; for, both 2 and 3 have $\alpha=1=\beta$.

\section{Step 8.}

The different arrays for $\delta^{\prime}$ are:

$\begin{array}{lllllllllllll}2 & 3 & 4 & 5 & 5 & 6 & & 2 & 3 & 4 & 5 & 5 & 6 \\ 1 & 1 & 2 & 4 & 3 & 4 & & 1 & 1 & 3 & 4 & 2 & 4\end{array}$


Step 9.

The corresponding tableaux are:

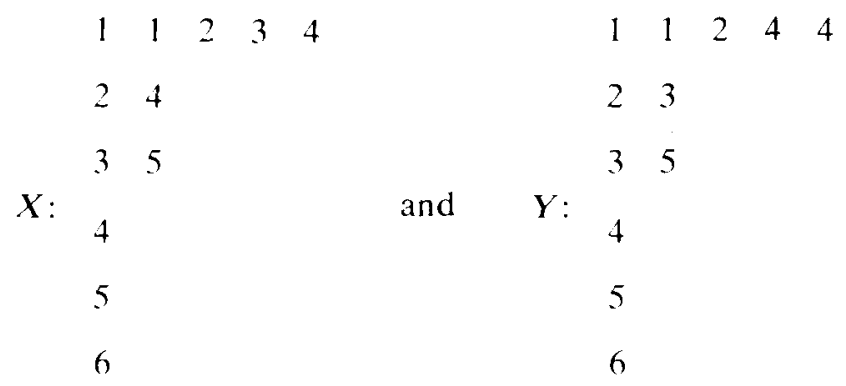

By the first letter sequence, $B=X$.

Definition 2. A tableau of shape-type $\alpha$ is said to be a topogeneous tableau if it is invariant for UNLABEL.

Clearly, the tableau $A$ of the previous example is not topogeneous. But it can be verified that the tableau $B$ is topogeneous. In fact, we have:

PROPOSITION 1. The range of the algorithm UNLABEL is the set of all topogeneous tableaux.

\section{Main theorem}

THEOREM 1. There is a constructive one-one correspondence between homeomorphism classes of non-discrete $T_{1}$-topologies on $X_{n}=\{1,2, \cdots, n\}$ and topogeneous tableaux of order $n$.

Proof. Given a topogeneous tableau of order $n$, form the associated two-line array by DELETE 3. The digraph $G$ corresponding to this array satisfies: (a) it is trivially transitive, because a topogeneous tableau is invariant for UNLABEL and in particular, for step 2 of UNLABEL; and (b) it has no cycles, because, in the array, $u_{k}>v_{k}$ for all $k$. Thus $G$ is an acyclic transgraph and as such (see Evans, Harary and Lynn (1967), Krishnamurthy (1975)) leads to a $T_{1}$-topology and also to its homeomorphism class.

Conversely, given any homeomorphism class $\mathscr{T}$ of nondiscrete $T_{0}$ topologies on $X_{n}$. take any such topology $\tau \in \mathscr{T}$. Construct the transgraph $G=G(\tau)$ associated with $\tau$ and remove all edges which are the result of transitivity; that is, whenever there is a chain $\{(a, b),(b, c), \cdots,(k, l)\}$ and also $(a, l)$ in the graph, remove $(a, l)$. Find the level decomposition $\eta\left(G^{\prime}\right)$ of the vertex set of the resulting graph $G^{\prime}=G^{\prime}(\tau)$ as in Krishnamurthy (1975). 
Order the level decomposition as in step 4 of UNLABEL. The resulting digraph, now called $G^{\prime \prime}(\tau)$, along with the level partition of $n$, is essentially a descriptor graph in the sense of Das (1973). In other words it satisfies and is defined by the following:

(DG1) There are $p_{i}$ vertices at level $i, i=1,2, \cdots \cdots, l$ and $p_{1}+p_{2}+\cdots+p_{1}=n$;

(DG2) The edges are always directed from 'higher' to 'lower' levels;

(DG3) Each vertex at level $i+1, i=1,2, \cdots, l-1$ is joined to at least one vertex at level $i$; and

(DG4) If $x$ is at level $i+1$ and $y$ is at level $j, j<i$, and there is a directed path from $x$ to $y$, then there is no edge $(x, y)$.

Note that DG2 implies no two vertices at the same level are joined. Isomorphism of descriptor graphs is defined in an obvious way by requiring the correspondence of vertices to preserve adjacencies as well as levels.

The two-line array corresponding to the descriptor graph so obtained can be put in standard form without affecting the level decomposition, because this is the effect of mimicking step 4 for $G^{\prime}(\tau)$. Applying steps 5 to 9 of UNLABEL to this array we get a topogeneous tableau. That the resulting tableau is independent of the choice of $\tau \in \mathscr{T}$ follows from the fact that homeomorphic topologies have isomorphic descriptor graphs and that the steps 5 to 9 of UNLABEL just relabel the vertices, within each level, of the descriptor graph in a canonical way. This completes the proof of the theorem.

\section{Topogeneous symmetric expressions}

The interest of this and the next section is to anatomize the enumeration of the homeomorphism classes in order to have a glimpse of a possible generating function for the same. With this end in view, we introduce

Definition 3. A Topogeneous Symmetric Expression (TSE, for short) (of order $n$ ) is a formal expression that arises by the application of steps 1 to 5 of UNLABEL on a topogeneous tableau (of order $n$ ).

Clearly, a TSE has properties (*) of step 6 of UNLABEL.

Knowing that topogeneous tableaux and certain canonically labelled descriptor graphs are in one-one correspondence, we see that every TSE arises from such descriptor graphs by formation of products of the type

$$
\prod_{i} x_{i}^{\alpha_{i}} \prod_{i} y_{i}^{\beta_{i}}
$$


where $x_{i}$ and $y_{i}$ stand for the vertex $i, \alpha_{i}$ (respectively, $\beta_{i}$ ) is the indegree (respectively, outdegree) of the vertex $i$. Once we have this interpretation of a TSE, all the properties stated in the Proposition below follow automatically:

Proposition 2. Every TSE of order $n$ is of the form $k_{\lambda} k_{\mu}$, where,

1. $n=p_{1}+p_{2}+\cdots+p_{1}$.

2. $d$ is an integer not less than $n-p_{1}$.

3. $\mu$ is a partition $\left(\beta_{i}\right)$ of $d$ into $n-p_{1}$ parts.

4. $\lambda$ is a partition $\left(\alpha_{i}\right)$ of $d$ into $q$ parts where $l-1 \leqq q \leqq n-p_{l}$.

Let $\zeta_{i}=\sum_{s=1}^{i} p_{s}, i=1,2, \cdots, l$ and $\zeta_{0}=0$. Then,

5. $k_{\lambda}=\prod_{i=1}^{\zeta_{1}-1} x_{i}^{\alpha_{i}}=\prod_{i=1}^{l-1} \xi_{i}$

with

$$
\xi_{l}=\prod_{i=\zeta_{1},-1}^{\zeta_{1}} x_{i}^{\alpha_{i}}, \quad j=1,2, \cdots, l-1 .
$$

6. For each $j=1,2, \cdots, l-1, a_{\xi_{1-1}+1}>0$.

7. $k_{\mu}=\prod_{i=\zeta_{1}+1}^{\zeta_{1}} y_{i}^{\beta_{i}}=\prod_{i=2}^{l} \psi_{i}$

with

$$
\psi_{i}=\prod_{i=\zeta_{j}}^{\zeta_{i}} y_{i+1}^{\beta_{i}}, j=2,3, \cdots l
$$

and all $\beta_{1}$ 's $>0$.

8. For each $j=1,2, \cdots, l-1$, the power of any $x$ in $\xi_{j}$ is not greater than

$$
p_{l}+\sum_{i=j+1}^{l-1} \max \left(p_{i}-1,1\right), \quad \text { if } \quad j \neq l-1
$$

and $p_{l}$, if $j=l-1$

9. For each $j=2,3, \cdots, l$ the power of any $y$ in $\psi_{i}$ is not greater than

$$
p_{1}+\sum_{i=2}^{i-1} \max \left(p_{i}-1,1\right), \quad \text { if } j>2
$$

and $p_{1}$, if $j=2$; and finally,

10. In every $\xi_{1}$ and $\psi_{j}, j=2,3, \cdots, l-1$,

$$
i>i^{\prime} \Rightarrow \alpha_{i} \leqq \alpha_{i}
$$


and $i>i^{\prime}, \alpha_{i}=\alpha_{i^{\prime}} \Rightarrow \beta_{i} \leqq \beta_{i^{\prime}} ;$ in $\xi_{1}, i>i^{\prime} \Rightarrow \alpha_{i} \leqq \alpha_{i^{\prime}}$ and in $\psi_{l}, i>i^{\prime} \Rightarrow \beta_{i} \leqq$ $\beta_{i}$.

Note that $d$ is the number of edges in the descriptor graph. It would be interesting to find an upper bound for $d$. Such an upper bound will help, perhaps, in characterising TSE's. In spite of the above properties being insufficient to characterise TSE's, we shall find in the next section they are sufficient for the purpose of enumeration, at least up to $n=5$, of unlabelled descriptor graphs with $n$ vertices.

\section{Three correspondences for enumeration purposes}

TheOREM 2. Let $X_{n}=\{1,2, \cdots, n\}$. Then, (a) with every homeomorphism class of $T_{0}$-topologies on $X_{n}$, there is associated a level partition of $n$;

(b) given a level partition of $n$, with every homeomorphism class of $T_{0}$-topologies on $X_{n}$ belonging to it, there is associated a TSE of order $n$; and

(c) given a level partition of $n$ and a TSE of order $n$, with every homeomorphism class of $T_{0}$-topologies on $X_{n}$ belonging to them, there is associated a tableaushape of shape-type $\alpha$.

Further, none of these three correspondences is one-one.

ProOf. The proof of (a) is already contained in the latter half of the proof of Theorem 1. Given a level partition, every unlabelled descriptor graph, labelled in an arbitrary way but still satisfying the requirement of step 4 of UNLABEL, finally gets a unique labelling on application of steps 5 to 9 of the algorithm. Correspondingly, a unique TSE can be constructed by application of steps 1 to 9 on the relabelled descriptor graph and its array. This proves (b). To prove (c) it is enough to note that every topogeneous tableau has a unique shape.

That none of these is a one-one correspondence can be seen from the following example. Consider the level partition 221 of 5 . Corresponding to this, with $d=4$, take the TSE,

$$
k_{211} k_{211}=x_{1}^{2} x_{2} x_{3} y_{3} y_{4} y_{5}^{2}
$$

There are two distinct topologies on $X_{5}$ corresponding to this, as shown below by their descriptor graphs, descriptor arrays and topogeneous tableaux. Note that the shapes of the tableaux are the same. 
Figure 1.

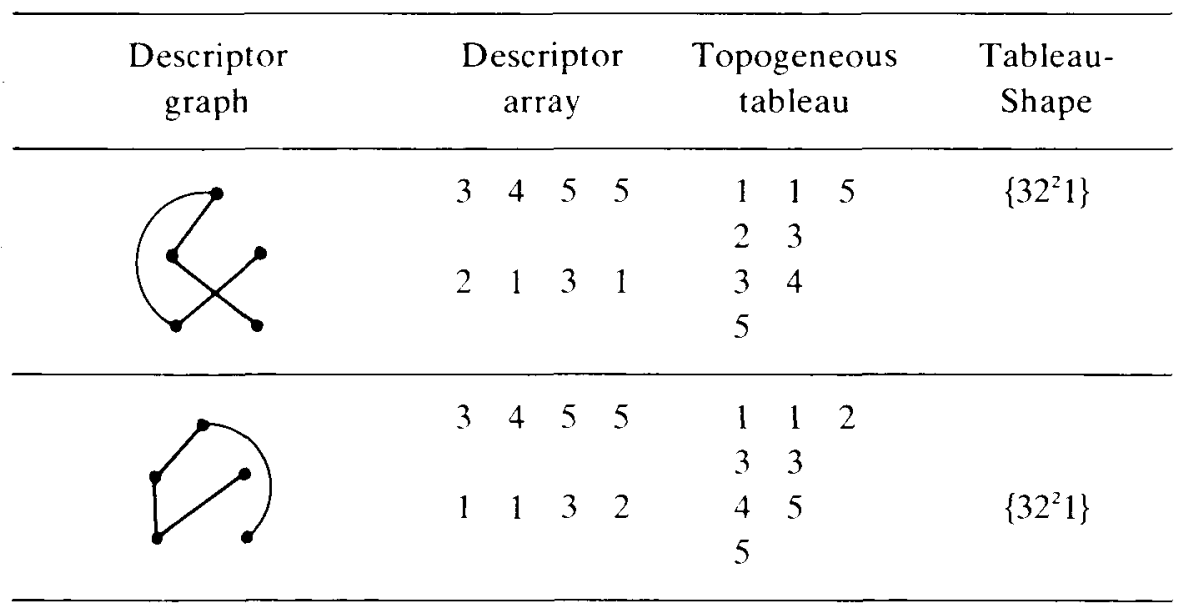

\section{Enumeration of homeomorphism classes of $T_{0}$-topologies}

The enumeration of unlabelled $T_{n}$-topologies can now be systematically done. Tables 1, 3, and 4 summarise these results showing the numbers $N_{\beta, \rho}$ of homeomorphism classes corresponding to the shape $\{\beta\}$ and level partition $\rho$, for $n=3,4$ and 5 respectively. These lead to the counting series $C_{3}, C_{4}, C_{5}$ of (2). In addition, the 15 topogeneous tableaux of order four are listed in Table 2. Finally, the results for $n=5$ are given in more detail, but, in order to conserve space, without the tableaux themselves, in Table 4 . The 62 descriptor graphs corresponding to the descriptor arrays of Table 4 are given serially in Table 4A. In all our tables the shape $\{\beta\}$ is written, for convenience, just $\beta$.

Table 1 .

\begin{tabular}{|c|c|c|c|}
\multicolumn{4}{c}{$N_{a, o}$ for $n=3$} \\
\cline { 2 - 4 } \multicolumn{1}{c|}{} & $d=1$ & $d=2$ & \\
\hline$\rho$ & $1^{2}$ & $21^{2}$ & $N_{p}$ \\
\hline 12 & & 1 & 1 \\
\hline 21 & 1 & 1 & 2 \\
\hline 111 & & 1 & 1 \\
\hline$N_{B}$ & 1 & 3 & 4 \\
\hline
\end{tabular}


Table 2 .

List of Topogeneous Tableaux of order four

\begin{tabular}{|c|c|c|c|c|c|c|c|c|c|c|c|}
\hline \multirow{4}{*}{\multicolumn{2}{|c|}{1}} & 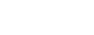 & 1 & \multicolumn{2}{|l|}{4} & 1 & 4 & 4 & 1 & 1 & 1 \\
\hline & & & 2 & & & 2 & & & 2 & & \\
\hline & & & 4 & & & 3 & & & 3 & & \\
\hline & & & & & & 4 & & & 4 & & \\
\hline & 1 & \multirow[t]{4}{*}{1} & 1 & \multirow{4}{*}{\multicolumn{2}{|c|}{2}} & 1 & 1 & & 1 & 1 & 4 \\
\hline & 3 & & 3 & & & 2 & 3 & & 2 & 2 & \\
\hline & 4 & & 4 & & & 3 & 4 & & 3 & 3 & \\
\hline & & & & & & & & & 4 & & \\
\hline & 1 & \multirow[t]{4}{*}{3} & 1 & \multirow[t]{4}{*}{3} & 3 & 1 & 2 & & 1 & 1 & 2 \\
\hline & 3 & & 2 & & & 3 & 3 & & 2 & & \\
\hline & 4 & & 3 & & & 4 & 4 & & 3 & & \\
\hline & & & 4 & & & & & & 4 & & \\
\hline & 1 & 12 & 1 & 2 & 2 & 1 & 2 & 3 & & & \\
\hline & 2 & 3 & 2 & & & 2 & & & & & \\
\hline & 3 & 4 & 3 & & & 3 & & & & & \\
\hline & 4 & & 4 & & & 4 & & & & & \\
\hline
\end{tabular}

Table 3.

$N_{\beta . \rho}$ for $n=4$

\begin{tabular}{|c|c|c|c|c|c|c|}
\hline & $d=1$ & $d=2$ & \multicolumn{2}{|c|}{$d=3$} & $d=4$ & \\
\hline$\rho$ & $1^{2}$ & $21^{2}$ & $31^{3}$ & $2^{3}$ & $32^{2} 1$ & $N$ \\
\hline 13 & & & 1 & & & 1 \\
\hline 31 & 1 & 1 & 1 & & & 3 \\
\hline 22 & & 2 & & 1 & 1 & 4 \\
\hline 112 & & & 1 & & & 1 \\
\hline 121 & & & 1 & & 1 & 2 \\
\hline 211 & & 1 & 1 & 1 & & 3 \\
\hline 1111 & & & 1 & & & 1 \\
\hline$N_{\beta}$ & 1 & 4 & 6 & 2 & 2 & 15 \\
\hline
\end{tabular}


Table 4.

Enumeration and Classification of Homeomorphism Classes of $T_{0}$-Topologies for $n=5$

(Note: There are 62 entries in this table. In order to economise space only sample entries are given. The complete table can be obtained from the author)

\begin{tabular}{|c|c|c|c|c|c|c|}
\hline \multirow{2}{*}{$\begin{array}{l}\text { Serial } \\
\text { Number }\end{array}$} & \multirow{2}{*}{$\begin{array}{l}\text { Level } \\
\text { parti- } \\
\text { tion }\end{array}$} & \multirow[b]{2}{*}{$d$} & \multicolumn{2}{|r|}{ TSE } & \multirow{2}{*}{$\begin{array}{c}\text { Descriptor } \\
\text { Array }\end{array}$} & \multirow{2}{*}{$\begin{array}{c}\text { Tableau- } \\
\text { Shape }\end{array}$} \\
\hline & & & $k_{\lambda} k_{\mu}$ & Actual expression & & \\
\hline 1 & 14 & 4 & $k_{4} k_{111}$ & $x_{1}^{4} y_{2} y_{3} y_{4} y_{5}$ & $\begin{array}{llll}2 & 3 & 4 & 5 \\
1 & 1 & 1 & 1\end{array}$ & $41^{4}$ \\
\hline 2 & 41 & 1 & $k_{1} k_{1}$ & $x_{1} y_{5}$ & $\begin{array}{l}5 \\
1\end{array}$ & $1^{2}$ \\
\hline 3 & & 2 & $k_{11} k_{2}$ & $x_{1} x_{2} y_{5}^{2}$ & $\begin{array}{ll}5 & 5 \\
2 & 1\end{array}$ & $21^{2}$ \\
\hline 4 & & 3 & $k_{111} k_{3}$ & $x_{1} x_{2} x_{3} y_{5}^{3}$ & $\begin{array}{lll}5 & 5 & 5 \\
3 & 2 & 1\end{array}$ & $31^{3}$ \\
\hline 5 & & 4 & $k_{1111} k_{4}$ & $x_{1} x_{2} x_{3} x_{4} y_{5}^{4}$ & $\begin{array}{llll}5 & 5 & 5 & 5 \\
4 & 3 & 2 & 1\end{array}$ & $41^{4}$ \\
\hline 21 & 212 & 3 & $k_{21} k_{111}$ & $x_{1} x_{3}^{2} y_{3} y_{4} y_{5}$ & $\begin{array}{lll}3 & 4 & 5 \\
1 & 3 & 3\end{array}$ & $31^{3}$ \\
\hline 22 & & 4 & $k_{211} k_{211}$ & $x_{1} x_{2} x_{3}^{2} y_{3} y_{4}^{2} y_{5}$ & $\begin{array}{llll}3 & 4 & 4 & 5 \\
1 & 3 & 2 & 3\end{array}$ & $32^{2} 1$ \\
\hline 23 & & & & $x_{1} x_{2} x_{3}^{2} y_{3}^{2} y_{4} y_{5}$ & $\begin{array}{llll}3 & 3 & 4 & 5 \\
2 & 1 & 3 & 3\end{array}$ & $41^{4}$ \\
\hline 24 & & 5 & $k_{221} k_{221}$ & $x_{1}^{2} x_{2} x_{3}^{2} y_{3} y_{4}^{2} y_{5}^{2}$ & $\begin{array}{lllll}3 & 4 & 4 & 5 & 5 \\
2 & 3 & 1 & 3 & 1\end{array}$ & $3^{2} 2^{2}$ \\
\hline 62 & 11111 & 4 & $k_{1111} k_{111}$ & $x_{1} x_{2} x_{3} x_{4} y_{2} y_{3} y_{4} y_{5}$ & $\begin{array}{llll}2 & 3 & 4 & 5 \\
1 & 2 & 3 & 4\end{array}$ & $41^{4}$ \\
\hline
\end{tabular}


Table $4 A$.

Descriptor graphs corresponding to the descriptor arrays of Table $4(n=5)$

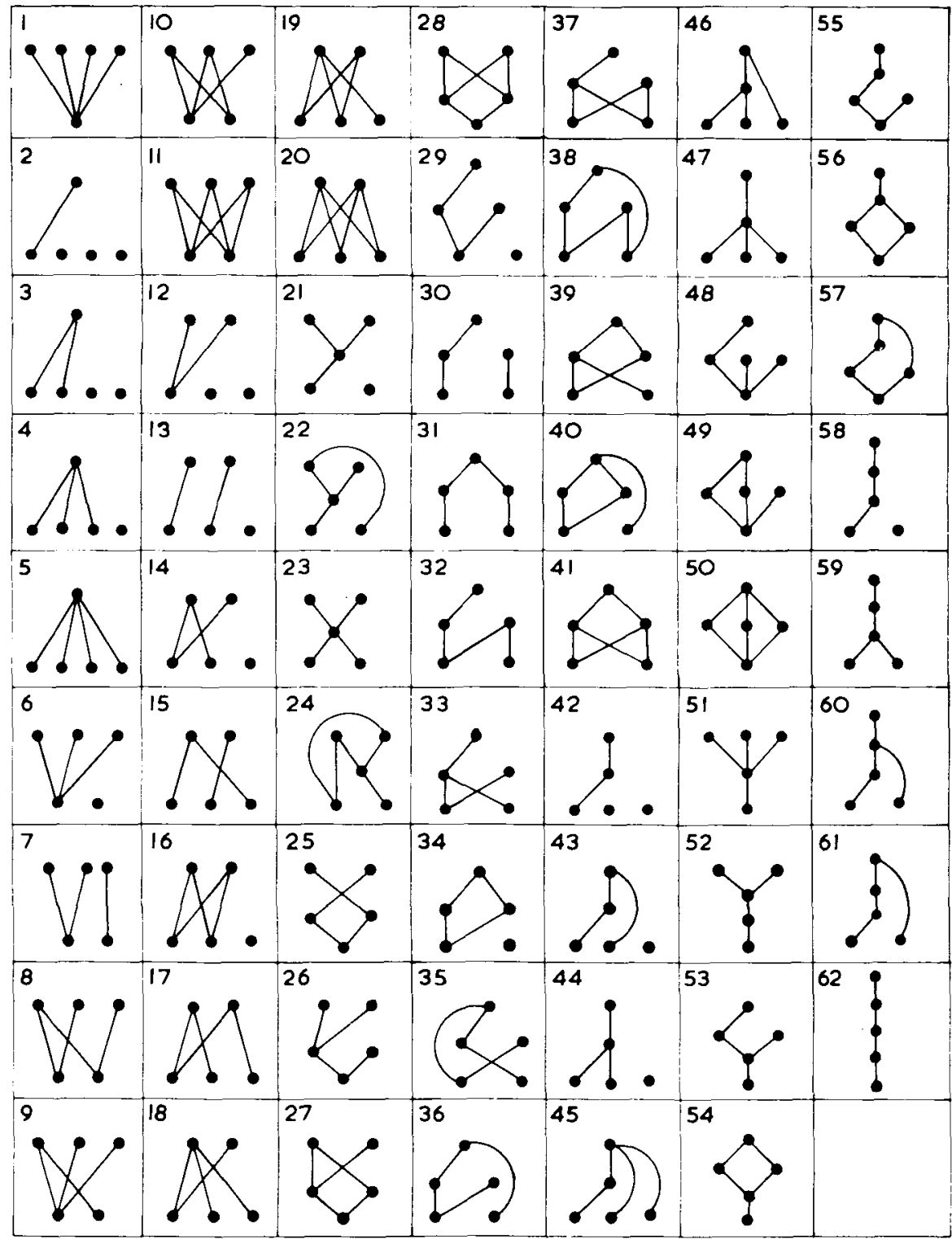


Table 5.

\begin{tabular}{|c|c|c|c|c|c|c|c|c|c|c|c|}
\hline \multicolumn{12}{|c|}{$N_{\beta, \rho}$ for $n=5$} \\
\hline \multirow[b]{2}{*}{$\rho$} & \multirow{2}{*}{$d=1$} & \multirow{2}{*}{$\begin{array}{l}d=2 \\
21^{2}\end{array}$} & \multicolumn{2}{|c|}{$d=3$} & \multicolumn{2}{|c|}{$d=4$} & \multicolumn{2}{|c|}{$d=5$} & \multicolumn{2}{|c|}{$d=6$} & \multirow[b]{2}{*}{$N_{p}$} \\
\hline & & & $31^{3}$ & $2^{3}$ & $32^{2} 1$ & $41^{4}$ & $3^{2} 2^{2}$ & $42^{2} 1^{2}$ & $3^{4}$ & $4.32^{2} 1$ & \\
\hline 14 & & & & & & 1 & & & & & 1 \\
\hline 41 & 1 & 1 & 1 & & & 1 & & & & & 4 \\
\hline 23 & & & 1 & 1 & 2 & & 1 & & 1 & & 6 \\
\hline 32 & & 2 & & 2 & 3 & & 1 & & & 1 & 9 \\
\hline 221 & & & 2 & & 6 & & 4 & & & 1 & 13 \\
\hline 212 & & & 1 & & 1 & 1 & 1 & & & & 4 \\
\hline 122 & & & & & 1 & 1 & & 1 & & 1 & 4 \\
\hline $.3 ! 1$ & & 1 & 1 & 1 & 2 & 1 & & & & & 6 \\
\hline 131 & & & & & & 1 & & I & & 1 & 3 \\
\hline 113 & & & & & & 1 & & & & & 1 \\
\hline 1112 & & & & & & 1 & & & & & 1 \\
\hline 1121 & & & & & & 1 & & 1 & & & 2 \\
\hline 1211 & & & & & & I & & 2 & & & 3 \\
\hline 2111 & & & 1 & & 2 & 1 & & & & & 4 \\
\hline 11111 & & & & & & 1 & & & & & 1 \\
\hline$N_{H}$ & 1 & 4 & 7 & 4 & 17 & 12 & 7 & 5 & 1 & 4 & 62 \\
\hline
\end{tabular}

\section{Enumeration of homeomorphism classes of non- $T_{0}$-topologies}

A transgraph represents a non- $T_{0}$ topology if and only if it contains a dwicycle (that is, cycles of length two) (see Evans, Harary and Lynn (1967)). Because of transitivity, the end points of any such dwicycle must either both be connected in the same direction or both be not connected, to any other chosen vertex. It follows that the end-points of a dwicycle are equivalent in the sense of the relation defined by the transgraph. Collapsing all such sets of equivalent vertices into single vertices we get a smaller number of vertices on which the resulting graph represents a $T_{0}$-topology. Thus in order to construct non- $T_{0}$ topologies on $X_{n}$ we proceed as follows:

Take any unordered partition $k_{1}+k_{2}+\cdots+k_{p}=n$ with at least one $k_{i}>1$. Whenever $k_{i}>1$, collapse a set $k_{i}$ of vertices of $X_{n}$ into a single vertex $x^{(i)}$. This gives us a set of $p$ vertices. Construct all non-homeomorphic $T_{0}$-topologies on this set of $p$ vertices by drawing their descriptor graphs. Treat each descriptor graph as a transgraph by drawing the arrows from higher to lower levels. Blow up each $x^{(i)}$ by recovering the original number of vertices which collapsed into it and connecting each pair of the $k_{i}$ equivalent 
Table 6.

Non- $T_{n}$-topologies on $X_{m}, n=2,3,4$

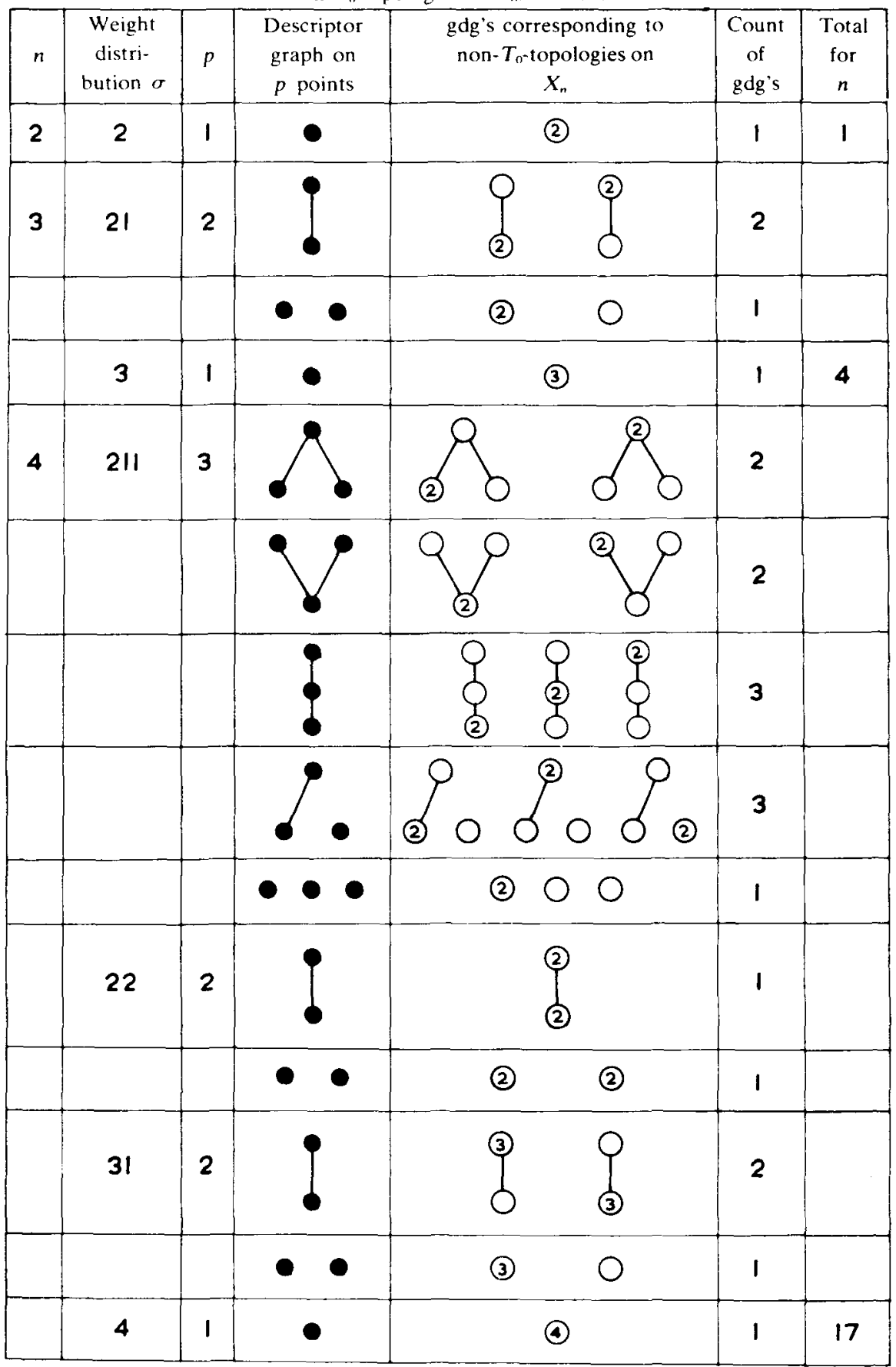


Table 7.

Values of $K_{a, p}$ for $n=5$ with $\sigma \neq 11111$.

\begin{tabular}{|c|c|c|c|c|c|c|}
\hline$\sigma$ & $p$ & $\rho$ & $\begin{array}{l}\text { No. of Descriptor } \\
\text { graphs on } p \text { ver- } \\
\text { tices with level } \\
\text { partition } \rho\end{array}$ & $K_{\infty, p}$ & $\begin{array}{l}\text { Count of } \\
\text { gdg's } \\
\text { for } \\
\text { given } \sigma\end{array}$ & $\begin{array}{c}\text { Total } \\
\text { for } \\
n\end{array}$ \\
\hline \multirow[t]{8}{*}{2111} & 4 & 1111 & 1 & 4 & & \\
\hline & & 211 & 3 & 11 & & \\
\hline & & 121 & 2 & 7 & & \\
\hline & & 112 & 1 & 3 & & \\
\hline & & 22 & 4 & 11 & & \\
\hline & & 31 & 3 & 8 & & \\
\hline & & 13 & 1 & 2 & & \\
\hline & & 4 & 1 & 1 & 47 & \\
\hline \multirow[t]{4}{*}{221} & 3 & 111 & 1 & 3 & & \\
\hline & & 21 & 2 & 5 & & \\
\hline & & 12 & 1 & 2 & & \\
\hline & & 3 & 1 & 1 & 11 & \\
\hline \multirow[t]{4}{*}{311} & 3 & 111 & 1 & 3 & & \\
\hline & & 21 & 2 & 5 & & \\
\hline & & 12 & 1 & 2 & & \\
\hline & & 3 & 1 & 1 & 11 & \\
\hline \multirow[t]{2}{*}{32} & 2 & 11 & 1 & 2 & & \\
\hline & & 2 & 1 & 1 & 3 & \\
\hline \multirow[t]{2}{*}{41} & 2 & 11 & 1 & 2 & & \\
\hline & & 2 & 1 & 1 & 3 & \\
\hline 5 & 1 & 1 & 1 & 1 & 1 & 76 \\
\hline
\end{tabular}

vertices by dwicycles. Complete all transitivities. The resulting transgraph represents a non- $T_{0}$ topology. Motivated by this discussion, we make the following

DEFINITION 4. A generalised descriptor graph $(g d g)$ is a graph which satisfies, in addition to DG1,DG2,DG3 and DG4, the following: To each vertex is attached a positive integer, called the weight of the vertex. If all weights are 1 , the gdg is the same as the descriptor graph.

DEFINITION 5. Two gdg's are isomorphic if there is a one-one correspondence of the sets of vertices, which preserves adjacencies, levels as well as weights.

Now the proof of the following theorem is clear. 
THEOREM 3. Two topologies on $X_{n}$ are homeomorphic if and only if their gdg's are isomorphic.

Table 6 gives the gdg's of the unlabelled non- $T_{n}$-topologies on $X_{n}$, $n=2,3,4$. In the gdg's the weight of a vertex, whenever it is greater than one, is indicated by a number placed within the circular dot standing for the vertex. Note that the descriptor graph corresponding to the discrete topology is a set of isolated points, all at level 1 .

Table 7 gives only a count of the corresponding information for $n=5$. Let $K_{\sigma, \rho}$ stand for the number of unlabelled topologies (=gdg's) on $n$ points corresponding to the unordered partition $\sigma$ (= weight distribution) and the ordered partition $\rho$ (=level partition) of $p \leqq n$. If $n=5$ and $\sigma=11111, K_{\sigma, p}$ is already given by $N_{\rho}$ in Table 5, except for $K_{1111,5}=1$ which is the count for the discrete topology. If any part of $\sigma$ is greater than one, then $K_{\text {o. }, p}$ counts non- $T_{0}$-topologies. This is the count that is given in Table 7 . Note that $\sum K_{\sigma, p}=1+62+76=139$, for $n=5$.

\section{References}

W. H. Burge (1974), 'Four correspondences between graphs and generalised young tableaux', $J$. Combinatorial Theory (A) 17, 12-30.

S. K. Das (1973), 'On the enumeration of finite maximal connected topologies', J. Combinatorial Theory 15, 184-199.

R. L. Davis (1953). 'The number of structures of finite relations', Proc. Amer. Math. Soc. 4, 486-495.

J. W. Evans. F. Harary and M. S. Lynn (1967). 'On the computer enumeration of finite topologies', Comm. ACM 10, 295-297.

F. Harary and E. M. Palmer (1962) Graphical enumeration (Academic Press, New York and London. 1973).

D. E. Knuth (1970), 'Permutations, matrices and generalised Young tableaux', Pacific J. Maths. 34. $709-727$.

V. Krishnamurthy (1966), 'On the number of topologies on a finite set'. Amer. Math. Monthly 73, $154-157$.

- (1975). Counting of finite topologies and a dissection of Stirling numbers of the second kind', Bull. Australian Math. Soc. 12, 111-124.

H. Sharp, Jr. (1966). 'Quasi-orderings and topologies on finite sets', Proc. Amer. Math. Soc. 17, $1.34+1349$.

M. Shiraki (1969). 'On finite topological spaces II'. Rep. Fac. Sci. Kagoshima Univ. 2, 1-15.

R. P. Stanley (1971). 'On the number of open sets of finite topologies'. J. Combinatorial Theory $10,74-79$.

Birla Institute of Technology and Science,

Pilani (Rajasthan), India. 